\title{
Acometimento orbitário na doença de Rosai-Dorfman
}

\author{
Orbital involvement in Rosai-Dorfman disease
}

Cristiana Dumaresq de Oliveira', Allan C. Pieroni Gonçalves², Frederico Castelo Moura³, Luiz Angelo Rossato ${ }^{1}$, Mário Luiz Ribeiro Monteiro ${ }^{4}$

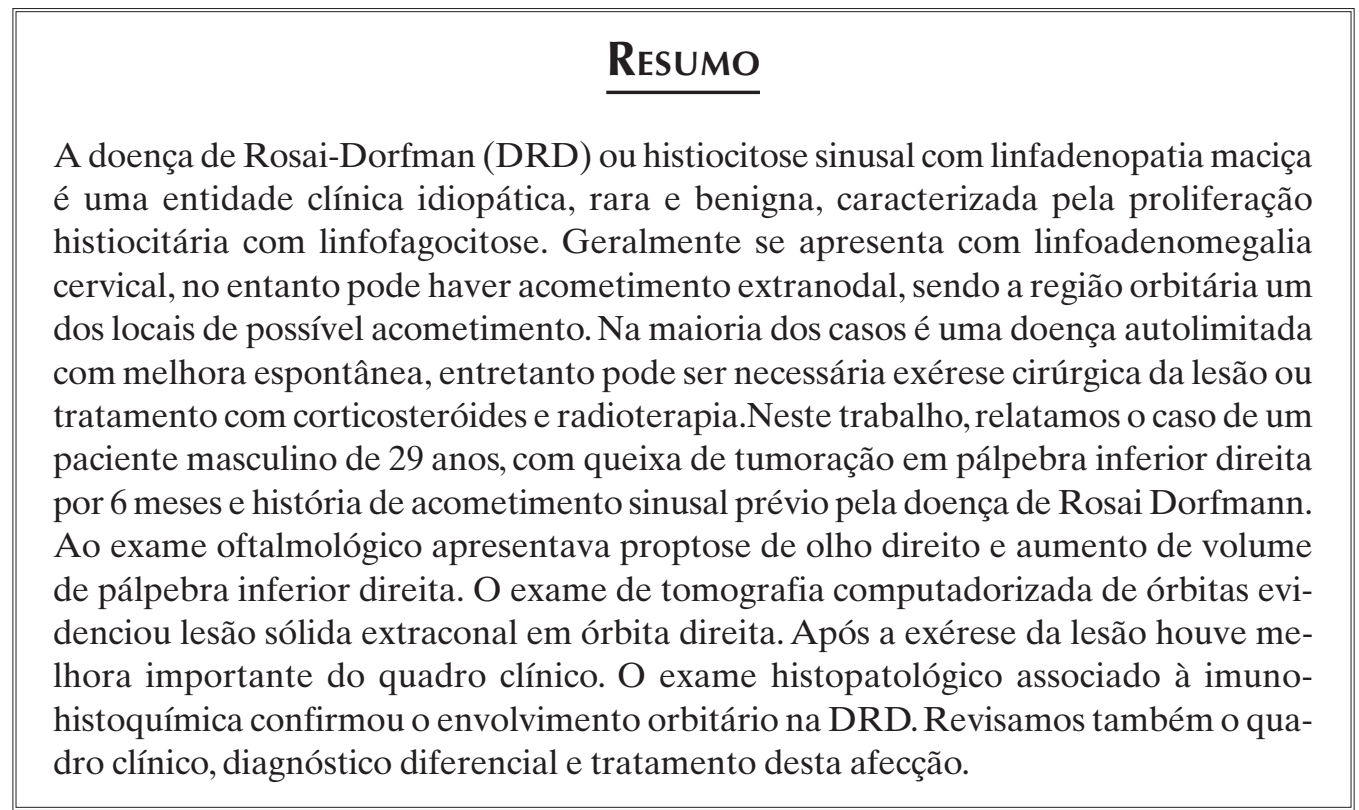

Descritores: Histiocitose sinus/diagnóstico; Histiocitose sinus/terapia; Exoftalmia; Linfonodos; Órbita; Seios paranasais; Relatos de casos

\footnotetext{
'Médico Residente do Hospital das Clínicas da Faculdade de Medicina da Universidade de São Paulo - USP - São Paulo (SP), Brasil; ${ }^{2}$ Pós-graduando (Doutorado), Disciplina de Oftalmologia da Faculdade de Medicina da Universidade de São Paulo - USP - São Paulo (SP), Brasil;

${ }^{3}$ Médico colaborador do Hospital das Clínicas da Faculdade de Medicina da Universidade de São Paulo - USP - São Paulo (SP), Brasil;

${ }^{4}$ Professor Associado, Disciplina de Oftalmologia da Faculdade de Medicina da Universidade de São Paulo - USP - São Paulo (SP), Brasil.
}

Disciplina de Oftalmologia da Faculdade de Medicina da Universidade de São Paulo - USP - São Paulo (SP), Brasil. 


\section{INTRODUÇÃO}

A doença de Rosai-Dorfman (DRD), também conhecida como histiocitose sinusal com linfadenopatia maciça, foi descrita primeiramente em 1969 por Rosai e Dorfman ${ }^{(1)}$ e se caracteriza do ponto de vista histopatológico por uma proliferação histiocitária associada à linfofagocitose (denominada emperipolese) ${ }^{(1-7)}$. É uma condição rara, de etiologia desconhecida que usualmente acomete os linfonodos e se manifesta por linfadenopatia cervical bilateral e indolor ${ }^{(3,6,8-11)}$. No entanto, o acometimento extranodal pode ocorrer sendo a órbita um dos locais acometi$\operatorname{dos}{ }^{(7,11-15)}$.

Quando existe acometimento orbitário, as manifestações incluem proptose, ptose, restrição de motilidade ocular, presença de massas conjuntivais e perda visual decorrente da infiltração linfoproliferativa dos tecidos moles da órbita e pálpebras ${ }^{(4,7,10,11,16-19)}$. Como a doença é bastante rara, pode haver confusão diagnóstica com outras afecções da órbita, levando a tratamentos inadequados. O objetivo deste trabalho é apresentar um caso de DRD com acometimento orbitário e revisar o quadro clínico, o diagnóstico diferencial e o tratamento desta afecção.

\section{Relato de caso}

Paciente de 29 anos, masculino, foi encaminhado ao ambulatório de órbita do Hospital das Clínicas da Faculdade de Medicina da USP, relatando tumoração indolor com aumento progressivo por 6 meses em região de pálpebra inferior direita. Referia tratamento otorrinolaringológico com diagnóstico de DRD e acometimento de seio maxilar direito, tendo realizado sete procedimentos cirúrgicos nos últimos 14 anos. Não apresentava adenomegalia cervical ou de outras cadeias, febre, anemia ou leucocitose.

Ao exame oftalmológico, apresentava acuidade visual normal, massa de consistência fibrosa na loja inferior da órbita direita com deslocamento superior do globo ocular (Figura 1). A exoftalmometria era de $19 \mathrm{~mm}$ à direita e $16 \mathrm{~mm}$ à esquerda. Os exames de reflexos fotomotores, motilidade ocular extrínseca, fundo de olho e tonometria eram normais. A tomografia computadorizada de órbitas evidenciou lesão sólida extraconal na região inferior da órbita, estendendo-se desde o assoalho orbitário até o globo ocular (Figura 2).

Foi realizada ressecção da lesão por uma orbitotomia anterior via transconjuntival com incisão no fórnice inferior. Foram retirados dois fragmentos
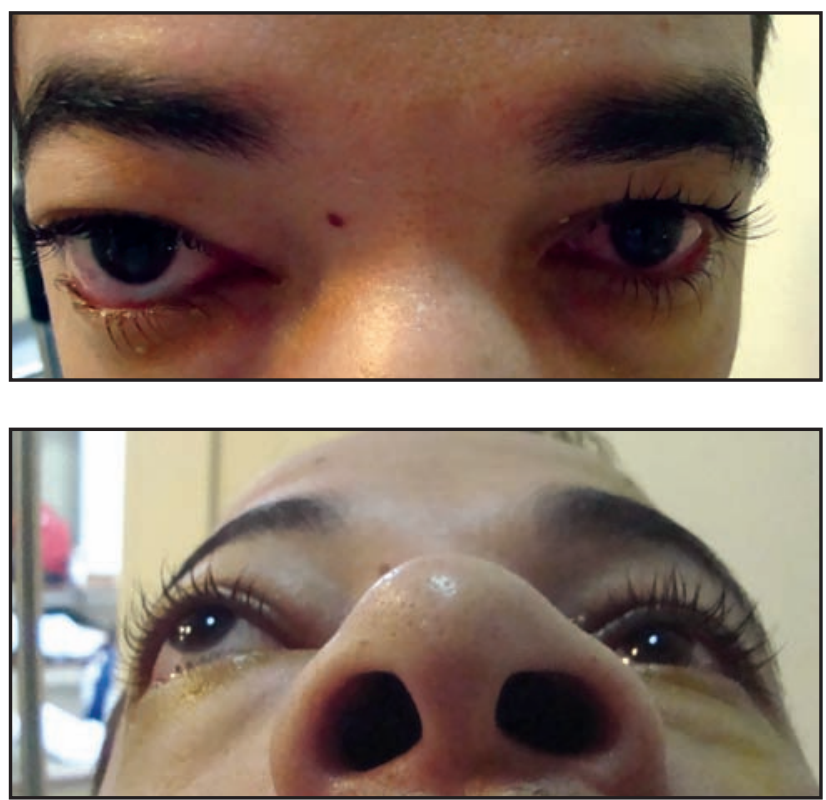

Figura 1: proptose e distopia superior em olho direito

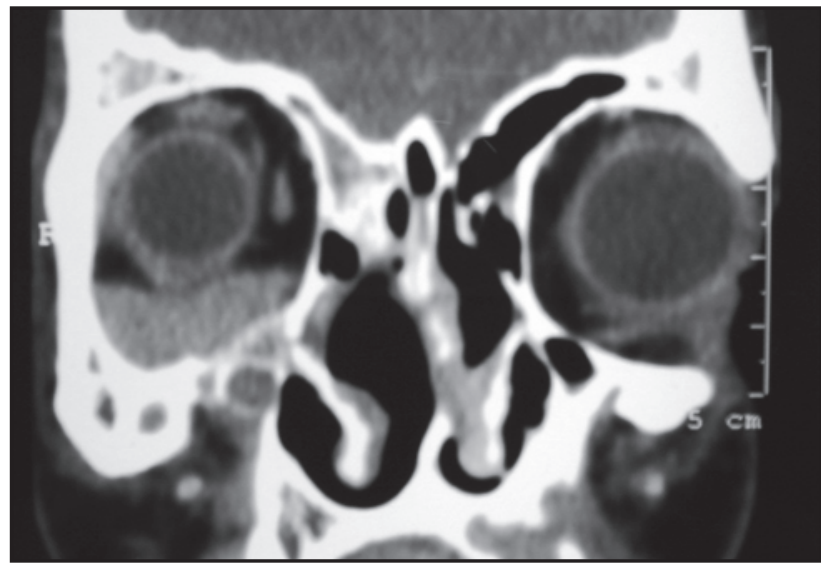

Figura 2: imagem de tomografia de órbitas em corte coronal, evidenciando massa extraconal inferior em órbita direita

medindo o primeiro $2,5 \times 1,0 \times 0,98 \mathrm{~cm}$ e o segundo $1,4 \mathrm{x}$ $0,9 \times 0,6 \mathrm{~cm}$. A análise histopatológica confirmou o diagnóstico de DRD, com presença de proliferação histiocitária e linfofagocitose. O estudo imunohistoquímico foi positivo para proteína S-100. (Figura 3 A e B). Após a cirurgia, o paciente evoluiu com melhora importante das alterações do exame no pós-operatório tendo se mantido desta forma em seguimento de dois anos (Figura 4).

\section{DiscusSÃo}

A DRD é uma condição rara que se caracteriza por proliferação histiocitária de natureza benigna e idiopática ${ }^{(1,2,5,8)}$. O vírus Epstein-Barr e o herpes vírus 

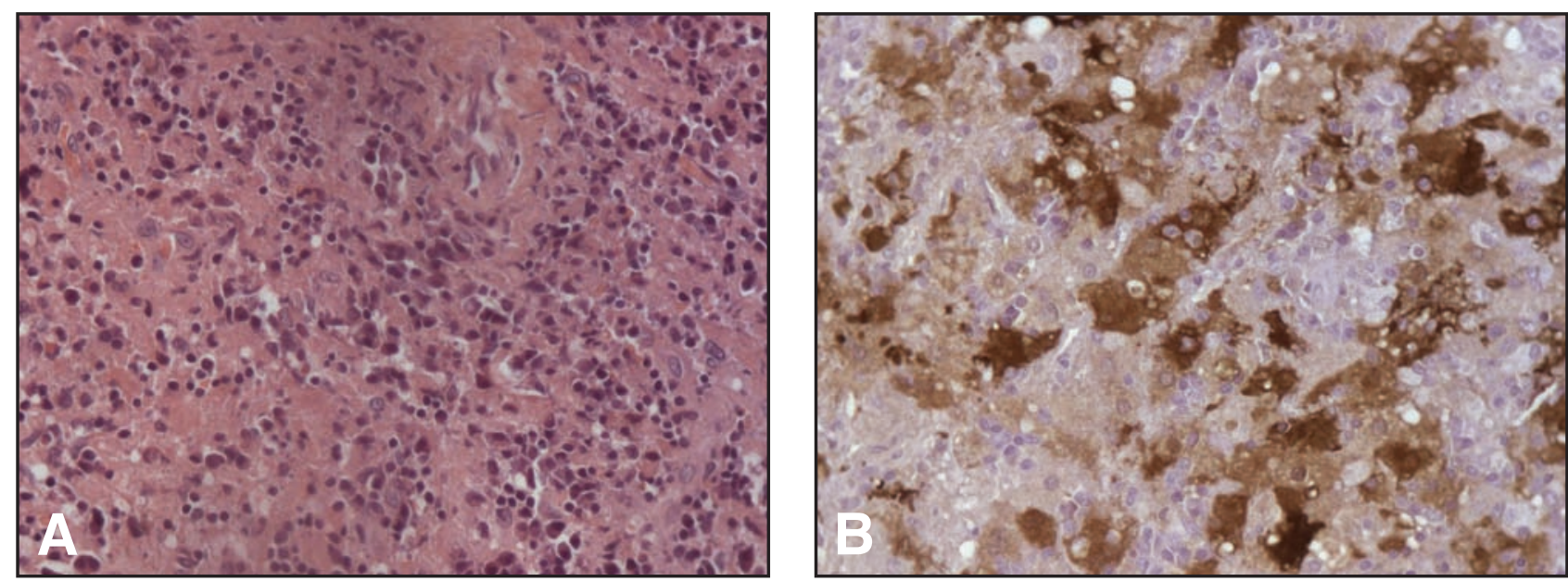

Figura 3: A - Histopatológico evidenciando infiltrado polimórfico de células inflamatórias, com linfócitos maduros, plasmócitos e histiócitos (x40, hematoxilina-eosina); B -Imuno-histoquímica positiva para proteína S-100 (x40)

humano tipo 6 foram isolados em alguns pacientes, mas ainda não foi confirmada uma associação com estes processos infecciosos e a etiologia permanece desconhecida ${ }^{(5,13,15,20)}$.

Em 80 a $90 \%$ dos casos a doença apresenta-se como linfadenopatia cervical importante bilateral e indolor ${ }^{(3,6,8-11,21)}$. A afecção é um pouco mais comum em homens $(58 \%)$ do que em mulheres ${ }^{(13,18,22,23)}$, com incidência similar entre brancos e negros, e menor em asiáticos ${ }^{(13,24)}$. Pode ocorrer em qualquer idade, apesar de ser mais frequente nas duas primeiras décadas de vida ${ }^{(5,11,13)}$, com idade média de aparecimento de 20,6 anos ${ }^{(22)}$. Linfonodos mediastinais, hílares, axilares e inguinais também podem estar envolvidos, porém em menor proporção ${ }^{(1)}$. Normalmente não há comprometimento hepático e esplênico ${ }^{(5,7,10)}$. Apesar do estado geral do paciente estar preservado, sinais sistêmicos de inflamação são comuns, como febre, anemia normocrômica, leucocitose com neutrofilia, aumento de velocidade de hemossedimentação e hipergamaglobulinemia policlonal ${ }^{(4,5,8)}$ o que pode contribuir na confusão com outras afecções inflamatórias e mesmo infecciosas.

O comprometimento extranodal é relatado em 25 a $43 \%$ dos casos ${ }^{(6,10-12,15,16,21,25)}$, usualmente com acometimento nodal associado ${ }^{(12)}$. Os locais mais comuns de acometimento extranodal, em ordem decrescente, são: pele, cavidade nasal e seios paranasais, pálpebras, órbita, ossos, glândula salivar e sistema nervoso central. Em alguns casos, como no paciente aqui apresentado, somente o acometimento extranodal é responsável pela manifestação da doença ${ }^{(26)}$ o que é uma situação bastante incomum ${ }^{(16)}$. Pacientes com diagnóstico de DRD em qualquer estágio devem ser submetidos à investigação sistêmica, principalmente em mediastino, retroperitônio,

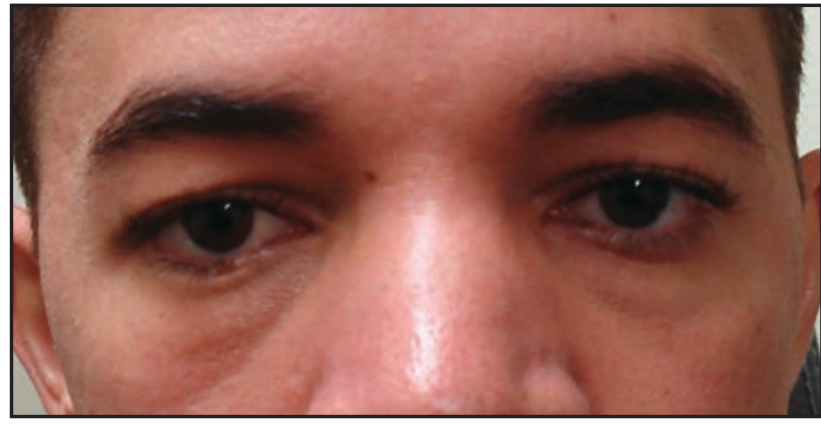

Figura 4: Pós-operatório

medula espinhal, sistema nervoso central, ossos e, nos homens, testículos para se determinar a extensão do acometimento da doença ${ }^{(18)}$.

O envolvimento oftalmológico na DRD ocorre em 8,5 a $11 \%$ dos $\operatorname{casos}^{(6,11,14,18,20,21,23)}$, mais frequentemente unilateral ${ }^{(6,14)}$ e se manifesta por: proptose, ptose, restrição de motilidade ocular, acometimento de glândula lacrimal, olho seco, neuropatia óptica compressiva e massas conjuntivais ${ }^{(4,7,10,14,18,27)}$. Raramente há comprometimento de estruturas intraoculares, mas uveíte pode ocorrer ${ }^{(4,9,10,14)}$. A proptose pode levar a ceratite por exposição, ulceração corneana e endoftalmite ${ }^{(9)}$. A maioria dos casos apresenta linfoproliferação de tecidos moles da órbita ${ }^{(4,7,10,11,16,18,20)}$, como tumor orbitário extraconal ${ }^{(9)}$. Pode haver extensão da lesão em direção a base do crânio, com destruição de estruturas ósseas vizinhas ${ }^{(13)}$. Em uma série de 113 casos, $11 \%$ apresentaram o acometimento oftalmológico como manifestação inicial ou principal da DRD ${ }^{(10)}$ e $54 \%$ dos pacientes com envolvimento oftalmológico apresentam outro local de comprometimento extranodal, principalmente na 
cavidade nasal ${ }^{(10,22)}$. No caso da órbita, tal local geralmente é a cavidade nasal e seios paranasais ${ }^{(22)}$.

A característica histológica da DRD é a dilatação subcapsular e medular do centro germinativo do linfonodo com proliferação histiocitária não neoplásica, além da presença de histiócitos com emperipolese ou linfofagocitose (histiócitos apresentando linfócitos fagocitados $)^{(1,4,6,7)}$ que coram com proteína S-100 na imuno-histoquímica ${ }^{(4,6,12)}$. Além dos histiócitos, o infiltrado é composto por linfócitos e células plasmáticas, que podem apresentar glóbulos eosinofílicos intracitoplasmáticos chamados corpúsculos de Russel ${ }^{(8,9,12)}$. Neutrófilos são raros, eosinófilos usualmente são ausentes e mitoses são extremamente raras, mesmo nas áreas de proliferação celular. Ocorre também fibrose capsular e perinodal extensas $^{(6,8,10)}$. O infiltrado extranodal, por sua vez, é composto por uma população de células similar ao nodal, apesar de ter fibrose mais intensa e menor proporção de histiócitos típicos e de emperipolese ${ }^{(12,18,22)}$. A doença é hoje considerada uma histiocitose não Langerhans por não apresentar grânulos de Birbeck e ser negativa para CD1a, o que permite diferenciá-la das demais histiocitoses ${ }^{(12,15,21)}$.

O diagnóstico diferencial da DRD deve incluir principalmente o linfoma de células B e de Burkitt, o sarcoma granulocítico, o rabdomiosarcoma e o neuroblastoma metastático. Tais desordens podem ser diferenciadas com base no curso clínico rapidamente progressivo, além dos estudos laboratoriais e proliferação celular com atipias ${ }^{(1,10,12)}$. A diferenciação com pseudotumor orbitário e xantogranuloma é possível através das análises histológica e imuno-histoquímica e também pela presença de dor mais frequente no pseudotumor orbitário. Nos casos de acometimento da órbita associado aos seios paranasais, como o paciente aqui apresentado, a doença de Rosai Dorfmann deve ser diferenciada também da granulomatose de Wegener $(28,29)$ e de tumores malignos de seios paranasais com invasão orbitária.

A DRD é considerada uma doença crônica, indolente e autolimitada ${ }^{(5,7,12,16)}$, com períodos de exacerbação e de remissão, podendo haver resolução espontânea em até $54 \%$ dos casos. A linfadenopatia tende a persistir por longos períodos antes da resolução ${ }^{(9)}$ e o envolvimento extranodal não altera o curso da doença ou prognóstico ${ }^{(13)}$. Os estágios da DRD são: remissão, recorrência, doença persistente, mas estável, forma progressiva primária e morte (por acometimento hepático, obstrução de via aérea, infecção ou desordem imunológica) ${ }^{(12,18,30)}$. Casos brandos sem comprometi- mento funcional ou estético podem ser apenas observados ${ }^{(7)}$. Se houver febre maior que $38^{\circ} \mathrm{C}$ sem infecção documentada e/ou aumento súbito de tamanhao linfonodal, deve-se iniciar o uso de corticosteróides ${ }^{(31)}$. Apesar de ser considerada refratária ao tratamento ${ }^{(6)} \mathrm{e}$ não ter o curso afetado pelos vários modos terapêuticos ${ }^{(8)}$, pacientes com sintomas e tumor de localização que comprometam a função de algum órgão podem ser submetidos à radioterapia, quimioterapia (ciclofosfamida, vincristina e mercaptopurina são os mais comumente usados ou corticoterapia sistêmica ${ }^{(4-6,31)}$. A remoção cirúrgica do tumor deve ser considerada para controlar manifestações locais ${ }^{(7,16)}$, como descompressão do trato respiratório superior, seios paranasais e órbitas ${ }^{(4,13,23)}$. A recorrência da lesão orbitária após exérese cirúrgica é considerada incomum, mas pode ocorrer. Nosso paciente mostra que a cirurgia pode ser útil no sentido de melhorar a disfunção causada pelo processo patológico.

Em conclusão, o caso aqui apresentado serve para enfatizar a importância de se incluir a DRD no diagnóstico diferencial das lesões orbitárias associadas ou não a acometimento de seios paranasais de tal forma a permitir um diagnóstico precoce e orientação terapêutica adequada.

\section{Abstract}

Rosai-Dorfman disease or sinus histiocytosis with massive lymphadenopathy is a rare idiopathic and benign clinical entity, characterized by histiocytic proliferation with linfofagocitosis. It usually presents with cervical lymphadenopathy although extranodal involvement may occur. The orbital region is one of the most common extranodal sites. It is usually a selflimiting disease with spontaneous resolution but surgical excision, corticosteroids and radiotherapy may be necessary. We describe the case of a 29-years-old male patient complaining of an orbital mass sensation for 6 months and a history of previous sinus involvement from Rosai-Dorfman disease. Ophthalmic examination showed proptosis of the right eye and swelling of right lower eyelid. Computed tomography of the orbits disclosed a solid extraconal lesion in the inferior right orbit. After surgical excision there was a significant clinical improvement. Histopathologic examination confirmed the diagnosis of Rosai-Dorfman disease. We also review the clinical picture and differential diagnosis of this condition.

Keywords: Histiocytosis, sinus/diagnosis; Histiocytosis, sinus/therapy; Exophthalmos; Lymph nodes; Orbit; Paranasal sinuses; Case reports 


\section{ReFERÊNCIAS}

1. Rosai J, Dorfman RF. Sinus histiocytosis with massive lymphadenopathy. A newly recognized benign clinicopathological entity. Arch Pathol. 1969;87(1):63-70.

2. Salmon JF, Duffield M. Sinus histiocytosis with massive lymphadenopathy. Am J Ophthalmol. 1989;107(5):549-50.

3. Brau RH, Sosa IJ, Marcial-Seoane MA. Sinus histiocytosis with massive lymphadenopathy (Rosai-Dorfman disease) and extranodal involvement of the orbit. P R Health Sci J. 1995;14(2):145-9.

4. Yip CC, Cheng CL, Poh WT, Choo CT. Orbital, adnexal, and unusual systemic involvement in Rosai-Dorfman disease. Ophthal Plast Reconstr Surg. 2002;18(3):223-7.

5. Khan R, Moriarty P, Kennedy S. Rosai Dorfman disease or sinus histiocytosis with massive lymphadenopathy of the orbit. Br J Ophthalmol. 2003;87(8):1054.

6. Wu SY, Ma L, Tsai YJ. Partial removal of orbital tumor in Rosai-Dorfman disease. Jpn J Ophthalmol. 2004;48(2):154-7.

7. Vemuganti GK, Naik MN, Honavar SG. Rosai dorfman disease of the orbit. J Hematol Oncol. 2008;1:7.

8. Rosai J, Dorfman RF. Sinus histiocytosis with massive lymphadenopathy: a pseudolymphomatous benign disorder. Analysis of 34 cases. Cancer. 1972;30(5):1174-88.

9. Friendly DS, Font RL, Rao NA. Orbital involvement in 'sinus' histiocytosis. A report of four cases. Arch Ophthalmol. 1977;95(11):2006-11

10. Foucar E, Rosai J, Dorfman RF. The ophthalmologic manifestations of sinus histiocytosis with massive lymphadenopathy. Am J Ophthalmol. 1979;87(3):354-67.

11. Prabhakaran VC, Bhatnagar A, Sandilla J, Olver J, Leibovitch I, Ghabrial R, et al. Orbital and adnexal Rosai-Dorfman disease. Orbit. 2008;27(5):356-62.

12. Wenig BM, Abbondanzo SL, Childers EL, Kapadia SB, Heffner DR. Extranodal sinus histiocytosis with massive lymphadenopathy (Rosai-Dorfman disease) of the head and neck. Hum Pathol. 1993;24(5):483-92.

13. Remadi S, Anagnostopoulou ID, Jlidi R, Cox JN, Seemayer TA. Extranodal Rosai-Dorfman disease in childhood. Pathol Res Pract. 1996;192(10):1007-15.

14. de Silva D, Joshi N. Rosai-Dorfman disease recurrence with bilateral orbital masses following immunosuppressant therapy. Orbit. 2005;24(1):51-3.

15. Hinduja A, Aguilar LG, Steineke T, Nochlin D, Landolfi JC. Rosai-Dorfman disease manifesting as intracranial and intraorbital lesion. J Neurooncol. 2009;92(1):117-20.

16. Vemuganti GK, Shekar CG, Indira K. Multifocal Rosai Dorfman disease of periorbital tissues spanning 15 years - a case report. Orbit. 2001;20(4):297-300.

17. Meyer CH, Sel S, Hörle S, Burg J, Kroll P. Rosai-Dorfman disease with bilateral serous retinal detachment. Arch Ophthalmol. 2003;121(5):733-5.

18. Mohadjer Y, Holds JB, Rootman J, Wilson MW, Gigantelli JW, Custer PL. The spectrum of orbital Rosai-Dorfman disease. Ophthal Plast Reconstr Surg. 2006;22(3):163-8.
19. Jogai S, Temmim L, Al-Baghli A, Adesina AO. Rosai-Dorfman disease presenting as bilateral eyelid swellings. Med Princ Pract. 2006;15(2):163-5.

20. Maheshwari R, Shekde S. Extranodal Rosai-Dorfman disease presenting as an isolated epibulbar mass. Indian $\mathbf{J}$ Ophthalmol. 2008;56(6):502-4.

21. Fu CH, Huang SF, Jung SM, Chin SC, Liao CT, Chen IH. Radiology quiz case 2. Extranodal Rosai-Dorfman disease involving the right orbit and nasal cavity. Arch Otolaryngol Head Neck Surg. 2009;135(3):317, 319

22. Foucar E, Rosai J, Dorfman R. Sinus histiocytosis with massive lymphadenopathy (Rosai-Dorfman disease): review of the entity. Semin Diagn Pathol. 1990;7(1):19-73.

23. Paire V, Bossard C, Vabres B, Weber M, Péchereau A. [Orbital locations in Rosai-Dorfman disease: a series of three consecutive cases]. J Fr Ophtalmol. 2008;31(7):673-82. French.

24. El-Banhawy OA, Farahat HG, El-Desoky I. Facial asymmetry with nasal and orbital involvement in a case of sinus histiocytosis with massive lymphadenopathy (Rosai-Dorfman disease). Int J Pediatr Otorhinolaryngol. 2005;69(8):1141-5.

25. Asai A, Matsutani M, Kohno T, Fujimaki T, Tanaka H, Kawaguchi K, et al. Leptomeningeal and orbital benign lymphophagocytic histiocytosis. Case report. J Neurosurg. 1988;69(4):610-2.

26. Foucar E, Rosai J, Dorfman RF. Sinus histiocytosis with massive lymphadenopathy. Arch Otolaryngol. 1978;104(12):687-93.

27. Albini TA, Evans M, See RF, Rao NA, Marback E, de Souza MM. Rosai-Dorfman disease: isolated epibulbar masses in two adult patients. Br J Ophthalmol. 2005;89(2):241-3.

28. Coppeto JR, Yamase H, Monteiro ML. Chronic ophthalmic Wegener's granulomatosis. J Clin Neuroophthalmol. 1985;5(1):17-25.

29. Monteiro ML, Borges WI, do Val Ferreira Ramos C, Lucato LT, Leite CC. Bilateral optic neuritis in wegener granulomatosis. J Neuroophthalmol. 2005;25(1):25-8.

30. Wang E, Anzai Y, Paulino A, Wong J. Rosai-Dorfman disease presenting with isolated bilateral orbital masses: report of two cases. AJNR Am J Neuroradiol. 2001;22(7):1386-8.

31. Pulsoni A, Anghel G, Falcucci P, Matera R, Pescarmona E, Ribersani M, et al. Treatment of sinus histiocytosis with massive lymphadenopathy (Rosai-Dorfman disease): report of a case and literature review. Am J Hematol. 2002;69(1):67-71. Review.
Endereço para correspondência:
Cristiana Dumaresq de Oliveira
Rua Teodoro Sampaio, no 363 - apto 708 - Pinheiros
São Paulo - (SP), Brasil
Tel: (11) 8604-2053
Email: crisdumaresq01@yahoo.com.br 